\title{
Édouard Montpetit
}

Économiste, Université de Montréal (1881-1954)

(1935)

\section{"L'avenir économique des Canadiens français."}

Un document produit en version numérique par Jean-Marie Tremblay, bénévole, professeur de sociologie retraité du Cégep de Chicoutimi

Courriel: jean-marie_tremblay@uqac.ca

Site web pédagogique : http://www.uqac.ca/jmt-sociologue/

Dans le cadre de: "Les classiques des sciences sociales"

Une bibliothèque numérique fondée et dirigée par Jean-Marie Tremblay, professeur de sociologie au Cégep de Chicoutimi

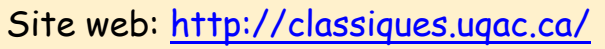

Une collection développée en collaboration avec la Bibliothèque

Paul-Émile-Boulet de l'Université du Québec à Chicoutimi

Site web: $\underline{h t p: / / b i b l i o t h e q u e . u q a c . c a l}$ 


\section{Politique d'utilisation de la bibliothèque des Classiques}

Toute reproduction et rediffusion de nos fichiers est interdite, même avec la mention de leur provenance, sans l'autorisation formelle, écrite, du fondateur des Classiques des sciences sociales, Jean-Marie Tremblay, sociologue.

Les fichiers des Classiques des sciences sociales ne peuvent sans autorisation formelle:

- être hébergés (en fichier ou page web, en totalité ou en partie) sur un serveur autre que celui des Classiques.

- servir de base de travail à un autre fichier modifié ensuite par tout autre moyen (couleur, police, mise en page, extraits, support, etc...),

Les fichiers (.html, .doc, .pdf, .rtf, .jpg, .gif) disponibles sur le site Les Classiques des sciences sociales sont la propriété des Classiques des sciences sociales, un organisme à but non lucratif composé exclusivement de bénévoles.

Ils sont disponibles pour une utilisation intellectuelle et personnelle et, en aucun cas, commerciale. Toute utilisation à des fins commerciales des fichiers sur ce site est strictement interdite et toute rediffusion est également strictement interdite.

L'accès à notre travail est libre et gratuit à tous les utilisateurs. C'est notre mission.

Jean-Marie Tremblay, sociologue

Fondateur et Président-directeur général, LES CLASSIQUES DES SCIENCES SOCIALES. 
Cette édition électronique a été réalisée par Jean-Marie Tremblay, bénévole, professeur de sociologie au Cégep de Chicoutimi à partir de:

\section{Édouard Montpetit}

"L'avenir économique des Canadiens français".

Un texte publié dans l'ouvrage sous la direction de Roger-J. Bédard, L'essor économique du Québec, pp. 494-502. Montréal : Librairie Beauchemin, 1969, 524 pp. Un article originalement publié dans Bulletin de la Chambre de Commerce de Montréal, février 1935, pp. 17-21.

Polices de caractères utilisée: Comic Sans, 10 points.

Édition électronique réalisée avec le traitement de textes Microsoft Word 2008 pour Macintosh.

Mise en page sur papier format : LETTRE US, $8.5^{\prime \prime} \times 11^{\prime \prime}$.

Édition numérique réalisée le 4 avril 2011 à Chicoutimi, Ville de Saguenay, Québec. 


\section{Édouard Montpetit}

"L'avenir économique des Canadiens français."

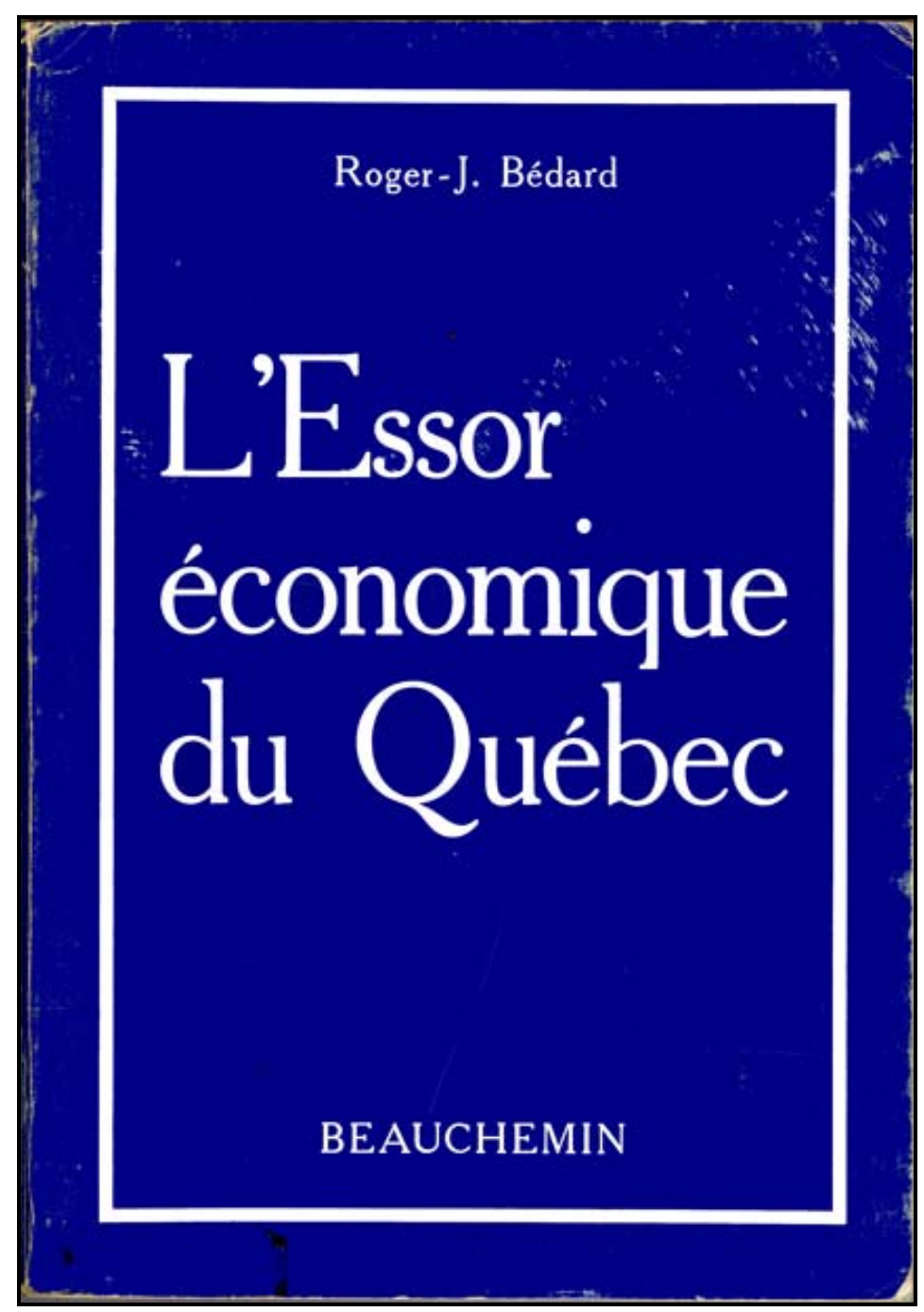

Un texte publié dans l'ouvrage sous la direction de Roger-J. Bédard, L'essor économique du Québec, pp. 494-502. Montréal : Librairie Beauchemin, 1969, 524 pp. Un article originalement publié dans Bulletin de la Chambre de Commerce de Montréal, février 1935, pp. 17-21. 


\section{Edouard Montpetit}

"L'avenir économique des Canadiens français". 1

Un texte publié dans l'ouvrage sous la direction de Roger-J. Bédard, L'essor économique du Québec, pp. 494-502. Montréal : Librairie Beauchemin, 1969, 524 pp. Un article originalement publié dans Bulletin de la Chambre de Commerce de Montréal, février 1935, pp. 17-21.

Le sujet que j'aborde ce soir est sorti, m'a-t-on dit, de vos délibérations: ce n'est pas moi qui l'ai choisi. C'est un sujet difficile et complexe. Il exige beaucoup d'objectivité de qui veut le traiter ou tente de le traiter.

Vous savez tout ce que je vais vous dire et je pense que nous sommes d'accord sur les quelques aspects de l'avenir de notre vie économique que je vais essayer de dégager. Mais il est bon sans doute pour le bien commun, de s'entretenir de ces sortes de choses.

J'ai demandé à un de mes collègues au moment d'entreprendre cette étude, ou plutôt cette ébauche: «Que pensez-vous de l'avenir économique des Canadiens français ? « «L plus clair que je sache, » $m^{\prime} a-t-i l$ répondu, « c'est que cet avenir peut être mauvais. " Ce mot m'a décidé. N'arriverais-je qu'à indiquer quelques écueils en éclairant un peu la voie, je serais satisfait.

Je remercie le président de la Chambre de Commerce junior de ses trop aimables paroles et je suis bien heureux d'être au milieu de vous pour aborder trop rapidement, il est vrai, une pareille question.

1 Texte d'une conférence prononcée devant les membres de la Chambre de Commerce junior. 


\section{I}

La première chose à considérer à mon sens, c'est que notre vie économique s'agite dans un milieu, dans ce que les Anglais appellent un environnement. La première connaissance que nous devons acquérir, est donc celle du milieu : le milieu physique avec ses richesses et ses pauvretés, ses influences passées et futures, afin que nous déterminions l'ensemble de notre action sur un territoire donné.

En second lieu, une fois que nous aurons connu ce territoire - je veux dire, une fois que nous en aurons pénétré le détail, dessiné la physionomie, une fois que nous saurons la valeur de nos vallées, les possibilités, comme on dit aujourd'hui, de nos montagnes, que nous saurons le cadre de notre activité - il faudra prendre conscience de [495] notre groupe ethnique par la philosophie de ses origines et de ses destinées, afin de cristalliser nos volontés. Connaissez-vous un congrès qui ait posé la question suivante: «Qu'est-ce qu'un Canadien français ? > Connaissez-vous même beaucoup de publications qui aient défini le Canadien français? Être Canadien, c'est être attaché à un territoire et être Français ce n'est pas être attaché à la France, mais c'est accepter de garder sur cette terre l'héritage français. Notre devoir est donc d'apprendre dans les écoles canadiennes l'attachement à notre terre et l'attachement à notre civilisation.

Notre groupe ethnique subit la concurrence d'autres groupes ethniques qui sont à côté de lui. Il peut leur emprunter des moyens d'action. Je crois même, Messieurs, que nous devons le faire, car si nous nous repliions sans cesse sur nous-mêmes je craindrais que nous n'aboutissions à l'infériorité. Mais si nous devons regarder autour de nous et consentir à demander aux autres des nouveaux moyens de survie, des moyens de développer notre économie, c'est à la condition essentielle que nous nous soyons fait d'abord un « coffre, » c'est-à-dire une physiologie forte, résistante et qui garde son caractère.

Pour fortifier nos traditions, il faut apprécier mieux les valeurs de civilisation que nous portons en nous-mêmes et que nous sommes loin d'avoir résolument exploitées, soit par insouciance, paresse, ou incompréhension. Nous nous persuaderons que ces valeurs de civilisation sont singulièrement précieuses. 
C'est un cliché funeste de toujours prétendre que ce qu'on est convenu d'appeler la race française ne peut pas réussir en affaires. La race française peut réussir en affaires. La France vous en fournirait beaucoup d'exemples. Je me rappelle avoir conduit dans un grand magasin de Paris un de mes jeunes compatriotes : le directeur avait bien voulu nous expliquer le fonctionnement de cet organisme économique et mon jeune ami était tout ébahi de ce qu'il voyait. Pendant les dernières vacances, alors que nous nous dirigions vers Saint-Malo, vers le pays de Jacques Cartier, j'ai longuement conversé avec le directeur des Chemins de Fer du Nord. Il m'a dit - je le savais déjà - que les chemins de fer européens sont souvent plus rapides que les nôtres et que les chemins de fer qui vont de Calais jusqu'à Paris, de Paris jusqu'à Bruxelles ou de Paris jusqu'à Rome sont peut-être les plus beaux que l'on connaisse au monde, il ne faut pas s'imaginer que l'on ne vit qu'à New-York, cette ville infernale où il y a tant de choses [496] qui ressemblent à des gares, et que lorsqu' on atteint Paris ou même d'autres villes beaucoup plus petites, on doive renoncer à tout confort. Je vous assure que le confort de Londres, le confort de Paris, le confort d'autres petites villes comme Reims est un confort tout à fait particulier, où on sent les subtilités de l'intelligence européenne. Voulez-vous questionner quelque ingénieur sur la valeur des formules du béton armé utilisées en France, il vous répondra que ces formules sont les plus sûres que l'on sache. Dois-je vous rappeler que bien souvent pendant le XIXe siècle, la France a été le banquier de Londres. Je dis, la France le banquier de Londres et non pas Londres le banquier de la France. Vous tous qui êtes Français vivant en Canadiens sur cette terre, que faites-vous! Nous portons en nous-mêmes des valeurs de civilisation que nous n'avons pas exploitées, je le répète: si seulement nous voulons nous tourner vers elles, les analyser, en vivre, les absorber, elles peuvent être l'occasion d'une vie économique extraordinaire.

Ce que j'admets, ce que j'accepte comme ligne de conduite pour ce groupe qui a une civilisation française, c'est ce que la France est un pays d'agriculteurs, d'artisans et de bourgeois, comme $M$. André Siegfried le répète un peu partout depuis plusieurs années. Je prétends que nous avons nous-mêmes tout le talent nécessaire, si nous voulons y joindre l'énergie, pour asseoir solidement notre propre économie sur ces trois éléments : le paysan, l'artisan et le bourgeois.

Cette civilisation, Messieurs, se traduit par la langue. Si les Canadiens français veulent affermir leur solidarité économique en constituant un groupe homogène et vigoureux, qu'ils pratiquent d'abord l'intégrité de leur langue. Rien ne vaut cette 
discipline. La langue, même dans le monde des affaires, même dans le rayon des intérêts matériels est un motif puissant au service du bien commun. Tant que nous n'aurons pas la fierté de la langue nous n'aurons pas les autres fiertés.

Il y deux jours, en quittant ma salle de cours où, sous la direction de mon directeur très sympathique, M. Henry Laureys, j'enseigne depuis vingt-cinq ans, je me dirigeais par le corridor (que quelques-uns d'entre vous connaissent par cœur) vers l'ascenseur. La porte de la salle de cours de mon collègue, Victor Barbeau, était ouverte et sur le tableau noir je relevai les deux questions d'examen que voici: $3 e$ préparatoire: Résumer sous forme de tableau I'histoire littéraire du XVIIe siècle: [497] 2 e préparatoire: Développer les raisons déjà données qui nous commandent de parler français. Pourquoi, Messieurs, en préparatoire, dans une école de commerce dirigée par un homme que la Belgique a formé, qui a été formé dans un pays où précisément les techniciens sont particulièrement recherchés, pourquoi des questions de français, pourquoi apprendre à des jeunes gens les raisons que nous avons de rester Français et de parler le français? Et pourquoi, avec l'École des Hautes Études commerciales, l'Université elle-même se penche-t-elle vers ce français pour le faire vivre et durer? Je sais que l'Université a encore beaucoup de progrès à faire et je sais aussi que ses reformes sont parfois malhabiles et qu'elles n'atteignent pas toujours jusqu' où elles voudraient atteindre. Mais ce que je sais, et ce que je suis prêt à prouver, ayant étudié cette question depuis un an et demi, c'est que, dans toutes ses facultés, depuis la faculté des Lettres jusqu'à l'École Polytechnique, jusqu'aux Sciences sociales et même - car c'est celle qui pourrait être la plus touchée - jusqu'à la faculté d'Art dentaire, I'Université de Montréal est au service de la survivance française et de la restauration française dans ce pays. Est-ce qu'on ne devrait pas enseigner l'anglais plus qu' on ne le fait à l'heure actuelle? Se préoccuper plutôt des disciplines techniques que de se préoccuper du français, véhicule de la pensée? $J$ 'ai pensé comme bien d'autres qu'il fallait savoir l'anglais, qu'il fallait dès le jeune âge apprendre l'anglais à nos enfants, mais j'en suis revenu! Il faut d'abord apprendre notre langue maternelle, il faut se former à notre discipline française et ce n'est que plus tard, lorsque le combat de la vie nous atteint, que nous devons nous tourner vers la connaissance de l'anglais, utilisant même l'anglais à parfaire notre vocabulaire français par le jeu des comparaisons.

Un jour, autour d'une même table, nous étions quelques-uns qui avions passé nos examens au barreau à peu près la même année. Nous ne nous étions pas parlé depuis 
bien des années et pourtant combien de pensées communes la vie avait fait descendre, avait cristallisées en nous-mêmes. Un Canadien français qui est devenu juge, fit un plaidoyer vigoureux en faveur de l'anglais. Au bout de la table, il y avait un homme qui, lui, a toujours vécu à Montréal dans les affaires. Quelle n'a pas été ma stupeur quand je l'ai entendu dire que l'anglais n'est pas nécessaire à ce point et qu'il ne voyait pas pourquoi on insistait. "Si nous savions nous comprendre, dit-il, nous unir, si nous savions prendre conscience de nos forces, si nous nous adressions à nos avocats, [498] à nos médecins, à nos ingénieurs, à nos commerçants, alors nous aurions tout un groupe prêt à collaborer à la grandeur du Canada, trouvant en lui-même de quoi se satisfaire largement, et dans son entourage propre de quoi constituer une vie économique.

Tout proche de la langue, il y a l'art. Je sais qu' on ouvre les yeux quand on parle d'art. Mais l'art est une manifestation par la beauté du même génie, de la même civilisation. Paul Anger demandait hier soir à l'Université de rééduquer le sens esthétique de notre population. Il a mille fois raison. L'Université a été longtemps toute seule à prêcher dans le désert de nos laideurs. Puis on a fondé l'École des BeauxArts dont l'influence se précise heureusement. Un collège classique, il y en a peutêtre d'autres, a inscrit à son programme de philosophie l'étude de l'art, I'histoire de l'art. L'art est même enseigné à l'école primaire, mais l'art ne dépasse pas l'école primaire, à cause du milieu un peu réfractaire. L'art est pour nous une discipline tout aussi bien que la langue; et l'art sera la source de toute une vie économique quand on se résoudra dans ce pays à reconnaître ses puissances, sa richesse, à admettre que l'art pour les Français que nous sommes est un singulier foyer de rayonnement. Par l'architecture, la sculpture, la musique, le théâtre, le cinéma, l'artisanat, quelles transformations vous pourriez faire subir à tout notre groupe qui est malheureusement satisfait d'à peu près et de boursoufflure? Ce progrès bienfaisant ne tient qu'à nous : il est à portée de notre main quand nous voudrons. Mussolini a sans doute rénové I'Italie, mais il a fait Rome. Vous avez porté à votre programme la construction d'une salle de spectacle et de concert. Excellente idée. Combien de choses il y aurait à rénover: le concert, les musiciens, toute la salle, les corridors, toute une foule qui serait vêtue, tous les taxis qui auraient amené cette foule. Mais que faisons-nous de votre âme française? À la condition de nous comprendre, d'aller jusqu'au fond de nous-mêmes, d'obéir à ce que nous sommes, tout l'avenir qu' une pareille transformation promet à une civilisation, nous l'aurons. 


\section{II}

Je crois que sur certains points nous sommes plus anglicisés qu'américanisés. Nous prenons des airs pour gourmander le sens pratique anglais, qui n'est pas fait pour nous, vous le pensez bien, et nous n'avons rien de plus pressé que d'imiter les Anglais dans ce qu'ils ont [499] peut-être de plus douteux ; l'empirisme. Notre grande erreur - et j'étais content tout à l'heure d'écouter ceux qui m'ont précédé notre grande erreur c'est de mépriser la théorie. Parce que Français nous devrions en vivre comme d'un élément logique et parce que, quoiqu' on fasse, la théorie est partout, même la mathématique dans le grand épanouissement de la musique.

Dès la première page de ses Sentiments et Coutumes, André Maurois inscrit cette pensée d'Auguste Comte: «Il faut rapprocher la sagesse théorique de l'admirable sagesse pratique». Voilà la vérité. Et tant que nous n'aurons pas reconnu cela, Messieurs, tant que nous n'aurons pas pratiqué cette théorie, et, si vous me passer cette expression théorisé cette pratique, nous continuerons de nous interroger sur notre avenir économique. Il nous faut une doctrine sur laquelle nous soyons d'accord. Je ne suis malheureusement pas de ceux qui peuvent la formuler. Je n'en ai ni le temps ni la capacité. Lorsque, Il y a quelques années, j'ai publié «Pour une doctrine», mon titre était un appel vers une doctrine, et je n'ai jamais eu la prétention d'en formuler une. Mais cette doctrine qui serait une pensée directrice, elle devrait inspirer notre politique - je prends le mot dans son sens le plus élevé, le plus large et cette politique devrait être une politique d'action, non pas une politique qui s'attarde dans des querelles qui sont toujours faciles, mais une politique constructive.

Notre vie économique, elle va malheureusement toute seule, au petit bonheur des intérêts, au gré des manoeuvres individuelles.

Nos institutions - les Chambres de Commerce, par exemple devraient s'y intéresser de très près afin d'en dégager les caractères spontanés et d'en organiser les rouages. Pas trop d'économie dirigée: mais une rénovation par la justice. Prenons les hommes comme ils sont plutôt que de les refaire par des mesures artificielles. Sachons ce que nous sommes capables de faire, ce que nous portons en nous-mêmes. L'avenir se moulera sur nos caractères spontanés. Pour organiser les rouages de la 
vie économique, on compte avec raison sur des associations comme la vôtre. Il ne s'agit pas de copier tel ou tel pays qu'on nous donne en exemple; nous en avons assez de rester nous-mêmes. Il faut diriger l'attention du public vers les questions économiques. On s'est trop peu occupé jusqu'ici de la vie économique, en mettant à part l'agriculture: on s'est désintéressé de notre industrie, de notre [500] commerce. Tâchons d'ouvrir la voie aux Canadiens français. Je me réjouis, pour ma part, du réveil qui se fait dans nos Chambres de commerce, qui se sont longtemps bornées à scruter des projets, qui prennent en main aujourd'hui les intérêts immédiats de nos entreprises.

Il est juste de dire que le sentiment général s'est modifié à l'endroit de l'économie nationale. Je ne me rappelle pas sans émoi l'accueil que fit, en 1910, un journal de Québec à celui qui osait, après bien d'autres, prêcher l'enrichissement aux Canadiens français. Bien des années passèrent avant qu'on s'avisât de considérer le problème canadien-français sous son aspect économique. Aujourd'hui, les circonstances nous imposent avec cruauté les conséquences de la crise que nous connaissons par expérience : on discute la monnaie, le change, l'impôt, les tarifs. Mais il reste quand même à dégager certaines vérités qui nous touchent. Vous pouvez vous y employer. L'association professionnelle, que l'on préconise avec raison, est le centre tout indiqué pour préparer l'opinion et agir avec discernement sur les pouvoirs publics.

Notre vie économique ne doit donc plus aller toute seule.

Dans le domaine de la production, l'étude de nos besoins m'a convaincu depuis longtemps que nous devons rester dans la moyenne et la petite industrie pour le moment. Nous avons un marché de trois millions de personnes qui s'habillent, s'alimentent et se logent. Il me semble que cela suffit.

En agriculture: l'économie familiale et la coopération. Le Danemark a trois millions cinq cent mille de population, la population même de la province de Québec. Le Danemark est une presqu'île que l'on traverse rapidement. Il nous donne le spectacle inoubliable de ses chalets luisant sous le soleil et de l'intensité de sa vie économique. Ce peuple qui a voulu s'instruire, coopérer, a fini par vaincre les marchés de l'Europe, le marché de Londres en particulier. Et nous qui avons une terre et une vallée, nous sommes encore là à nous demander ce que nous pouvons faire pour le réveil de l'agriculture. Il y a sûrement quelque chose qui ne marche pas quelque part! 
C'est dans le commerce que s'énonce aujourd hui la théorie de «l'achat chez les nôtres». L'Église catholique nous enseigne que tout homme a des devoirs et des droits. Les hommes, dans le domaine économique, ont aussi des devoirs et des droits. Voici une parole d'un [501] Anglo-Saxon, M.P. Ashley Cooper, qui disait devant une Association de commerçants de langue anglaise : « Le client est notre maître 》. Combien des nôtres admettent cette théorie, comprennent que la clientèle exige qu'on lui livre ce dont elle a besoin? L'intérêt du fournisseur est de satisfaire l'acheteur. Le sentiment seul ne suffit pas. il a la peau tendre et le cœur vif. Lorsque vous vous présentez chez un de vos compatriotes pour acheter quelque chose et qu'il vous répond: nous ne l'avons pas, le sentiment est définitivement vaincu. Il est arrivé à la femme d'un de mes collègues de demander dans un magasin tenu par un des nôtres: «Mademoiselle, auriez-vous l'amabilité de me dire laquelle de ces deux étoffes est de meilleure qualité » et de recevoir cette réponse: «Essayez-les, vous verrez ben ». Le sentiment cette fois est vite étouffé, et il se dirige ailleurs. L'achat chez les nôtres, c'est une formule légitime, mais vous ne devez pas oublier que le fournisseur a d'abord des devoirs. Je crois aussi que le fournisseur a le devoir de faire l'éducation de la clientèle, de lui faire comprendre l'intérêt de tous, le bien commun. Le fournisseur peut parfaitement former le goût du public. On me demande parfois: quel traité d'économie politique conseillez-vous? Je réponds: La grande rue. Allez vers l'Est, regardez les montres, les étalages, tout ce qui est offert au public, et vous comprendrez ce que nous avons encore à faire, non seulement pour répondre aux désirs de la clientèle, mais aussi pour satisfaire le goût et la beauté.

Nous avons aussi le devoir de nous associer. B. Ashley Cooper disait encore : « Il faut coopérer, il faut se solidariser». Il ajoutait qu'il n'est pas besoin pour cela de renoncer à sa personnalité. Comme nous sommes individualistes! Je ne veux pas dire que I'Anglo-Saxon ne soit pas individualiste. Il aime bien vivre chez lui, dans son home, il n'aime pas beaucoup qu' on le heurte. Il est très discret sur tout ce qui le concerne. Quand il a dit: It's a personal matter, il a tout dit. Mais à peine est-il sorti de cette vie première qu'il devient un coopérateur, il a dans l'esprit le team work. Apprenons de lui le sens de la solidarité, de la cohésion sans quoi nous n'arriverons à rien.

L'homme a aussi des droits, indubitablement. D'abord, celui de vivre; et d'accomplir notre fin, qui n'est ni la misère, ni le désintéressement devant le succès des autres. Ceux qui demandent que l'on achète des produits de l'Empire, ou des mar- 
chandises sorties des usines du [502] Canada font-ils autre chose que ceux qui adjurent les Canadiens français d'encourager leurs compatriotes? Je le répète cependant : n'en faisons pas une simple question de sentiment. C'est notre intérêt, l'intérêt général qui est en jeu. On ne fait pas un peuple fort avec des éléments faibles, même en les additionnant.

Le producteur, le commerçant ont des droits, que les théoriciens n'ont pas cessé de formuler et de défendre, depuis vingt-cinq ans que fonctionnent nos écoles spécialisées. À la tête de nos institutions, - Chambres de Commerce, Association des Marchands de détail, Hommes d'affaires, Ligue des propriétaires -, vous reconnaîtrez des diplômés, qui ont été formés dans l'intention de fournir des hommes à la vie économique. Les théoriciens ont travaillé d'accord avec la pratique, qui les a toujours guidés, et sauf autre ambition que celle d'accomplir de leur mieux une tâche souvent ingrate.

Des droits : ceux des individus, ceux de la nation. Cela nous ramène aussi à l'école. C'est l'école qui finira par nous donner des générations nouvelles possédant enfin! - l'intelligence de nos valeurs, de nos forces, le souci de l'action commune épaule à épaule, le respect de nos disciplines ethniques, la connaissance raisonnée des sciences économiques. C'est l'école dirigée qui nous permettra de réaliser un jour sur cette terre, sous le drapeau britannique, une France digne de celle qui nous a donné le jour.

Nous avons tout ce qu'il faut pour réussir, sauf la volonté.

Plusieurs des nôtres ont fondé des entreprises qui passent, les unes après les autres, aux mains des étrangers. Que font donc les fils de nos pionniers?

Plusieurs des nôtres, par la qualité et la nouveauté de leurs produits, ont même conquis la clientèle anglaise. Que ne les imitons-nous?

«Toujours protégés par des légistes et déterminés par des parleurs », selon le mot de Maurice Barrès, - qu'attendons-nous pour agir? 